\title{
LA VIOLENCIA DOMÉSTICA Y LA SOCIEDAD
}

Julio Munizaga B.

Consignamos aquí algunos puntos de tipo teórico y metodológico, interesantes de tener en cuenta, tanto para el lego como para el estudioso: sobre el tema de la violación doméstica y la sociedad.

La familia, desde que existe, ha estado sometida a múltiples presiones; sin embargo, la idea general de todos los teóricos y estudiosos de la familia concreta, indica que no está en vías de desaparecer; ni por los cambios en la tecnología y la modernización, ni tampoco por las situaciones que en estos momentos nos preocupan. Estamos mirando el problema como algo muy significativo, sin perjuicio de lo cual no hay que asignarle el timbre de crisis absoluta, sino intentar ver cuáles pueden ser los mecanismos que permitan controlar o minimizar esta situación, ya que cualquier situación emergente tiende a ser considerado como crítica, sin prever su efecto ni conocer su dimensión.

El segundo tema muy ligado a ésta, es pensar que la violencia intrafamiliar es un hecho nuevo, olvidando que cuando surge un fenómeno, la primera tendencia es pensar que es un fenómeno de ahora, y que si en el pasado hubo algo de ello, ese pasado, de alguna manera, tuvo una expresión de menor cuantía. Éstas son observaciones metodológicas válidas para todos los fenómenos sociales.

¿Qué pasa hoy? No tenemos suficiente información sobre el pasado, y menos todavía, en temas como éste. Recordemos que la familia era una caja de vida secreta, impenetrable; pero que de alguna manera, por los cambios en la forma de agrupación de las viviendas, el propio proceso de urbanización, los mecanismos de comunicación y de participación social, ha ido permitiendo que esta caja, impenetrable y secreta en muchos aspectos, se vaya abriendo, lo que ha ido permitiendo aproximaciones a la realidad del problema. En la medida que haya estudios más a 
fondo de la vida familiar, indudablemente que las cifras y estadisticas sobre violencia más nos van a alarmar.

En tercer lugar, nosotros estamos todos involucrados en el problema, porque todos tenemos familia; lo que hace difícil lograr la objetividad necesaria. Además un fenómeno multicausal, de causas heterogéneas y complejas, no debe mirarse con ángulos unicausales; visto como producto solamente del machismo, o desde el punto de vista de un movimiento feminista. Esto es mucho más complejo que eso.

Muy brevemente cito una observación de Ch. F. Thwing y C.E.R. Thwing, en el año 1887. "Los últimos cincuenta años han transformado, al parecer, las relaciones matrimoniales permanentes, para toda la vida, en otras que sólo se mantienen mientras resultan convenientes a las partes. El cambio, producido con rapidez, es tan revolucionario que afecta a las bases de la sociedad humana. Existe el convencimiento de que el matrimonio no es más que un contrato de carácter civil que al igual que otros contratos se establece por placer y conveniencia de las dos partes y, como en otros, se rescinde cuando ya no se obtiene más placer ni conveniencia" ${ }^{1}$. Si así era percibido, hace prácticamente 100 años atrás, ya existía una fundada noción de crisis; pero los estudiosos del tema piensan que la familia no va a desaparecer porque está hecha a la medida de ciertas necesidades únicas del hombre.

Debemos recordar algunos antecedentes de orden general, macrosocietal, de nuestra sociedad, $y$ el problema en Chile, donde somos aproximadamente doce millones quinientos mil seres humanos, que estamos agrupados en dos millones y medio de familias.

Por lo tanto, hay dos millones y medio de "espacios" donde esta "crisis" se produce o hay riesgo de que se produzca, y más o menos hacinadas en dos millones cincuenta mil viviendas de distintas características. Debemos observar, sin precipitarnos, lo que verdaderamente ocurre en materia de violencia intrafamiliar en estos 2,5 millones de "espacios" que son cambiantes, día a día, por el efecto de múltiples variables.

Hay otros dos indicadores que estimo que tienen incidencia con la estructura de la familia chilena y la estructura de la violencia intrafamiliar. Uno de éstos es el indice de masculinidad: en los grupos de edades de cero a cuatro años, hay 103,6 hombres por cada 100 mujeres; en el grupo de los 30 a 34 años, hay 99,8 hombres por cada cien mujeres; a la edad de los 40 años, se produce un equilibrio, cien contra cien; de ahí en adelante, la proporción masculina empieza a disminuir en forma vertiginosa hasta llegar a 58,9 hombres por cada 100 mujeres a la edad de ochenta años ${ }^{2}$.

En Goode, William J. La crisis de la institución familiar, Salvat, Madrid, 1975.

Instituto Nacional de Estadísticas y Censos de Chile, 1986. 
Esta situación tiene estrecha relación con el ejercicio de los roles familiares, con la presión, con la socialización. Hay un gráfico que ilustra expectativas de vida del hombre y mujer chilenos elaborado por CELADE del año 1950 al 2025, la expectativa. de vida del hombre chileno será de 58 años, y la expectativa de vida de la mujer dé 77 a 78 años. Hay una diferencia prácticamente de 18 a 19 años. Ésta es una característica estructural que no se puede desconocer o no tener presente en materias de planificación nacional.

Esto significa que parte de los problemas que nosotros estamos viviendo, van a ser influidos y manejados más por mujeres y por una menor cantidad de hombres. Es un asunto que está determinado no solamente por las reglas de la genética, también por la presión sociocultural sobre el hombre. Esto se da en otras partes del mundo; pero la diferencia es menor. El hombre parece que vive menos porque está sometido a una mayor cantidad de estrés. Por lo menos en todas las indagaciones que se conocen en el mundo occidental.

$Y$ otro dato que quiero recordar de paso es que tenemos cerca de unos tres millones seiscientos mil educandos, vamos a ver, después, por qué lo nombro.

Una consideración o premisa de orden biológico, es que la violencia y la fuerza es un ingrediente natural en la vida del hombre; está expreso o latente en todas sus manifestaciones. Muchos expertos en conducta humana estiman que si los seres vivos, por lo menos los vertebrados, no tienen una cantidad de fuerza que les permita sobreponerse a las condiciones duras del medio, desaparecen. Y éste ha sido un eterno problema del hombre, según las interpretaciones que se han dado de si es más materia o más espíritu, cómo controlar estas fuerzas, impulsos, desbordes, instintos; en el fondo: cómo controlar, un poco, la fuerza excesiva o desmedida.

Otra consideración es que tenemos que ubicar el fenómeno de la violencia familiar en el marco de la teoría interaccionista: la mayor cantidad de agresiones ocurre el día sábado, la mayor cantidad de agresiones ocurre en la mañana, o en la tarde, en los puntos de encuentro. El conflicto, la violencia y la fuerza se ejercen entre gentes que se conocen en la interacción $y$, desde este punto de vista, no olvidemos y analicemos que en nuestras propias vidas están ocurriendo una enorme cantidad de situaciones a las cuales estamos aplicando normas y valores, están surgiendo amores y odios. Es natural, y así lo dicen las estadísticas, que la mayor cantidad de agresiones tanto corporales o de fuerte presión psíquica, en general ocurren, un $75 \%$ entre conocidos y sólo en un $25 \%$ entre desconocidos.

Habitualmente aparecen en nuestra prensa, robos o crímenes... era un sobrino, un nieto, un allegado, un hombre que tenía alguna vinculación con el conocido; la familia es un grupo de conocidos ${ }^{3}$.

En Chile: Retamal, C. Pedro, 1987. Consideraciones sobre homicidio y esquizofrenia. En: Revista de Psiquiatría, órgano oficial del Hospital Psiquiátrico Dr. José Horwitz B. (pp. 61-66). Año ॥, N 12 - 
En este esquema, nosotros aplicamos una máxima de la antropología y de la sociología popular: "pueblo chico infierno grande". Y aquí tendríamos que homologar que la familia es una especie de sociedad pequeña, en donde nos estamos viendo todos los días a toda hora y con la posibilidad de influirnos y disputarnos el espacio, el silencio, el privilegio de estar solos o de estar acompañados, etc. El vivir en familia, implica un esfuerzo máximo de control sobre estos estados o impulsos naturales que muchas veces nos hacen reaccionar fuertemente.

Otra cosa que debemos recordar, es que la familia no se puede mirar como a un pez disecado; es un cuerpo vivo, esencialmente un grupo donde las relaciones son primarias. ¿En qué término son primarias? Porque son esencialmente emotivas, afectivas, el juicio y la razón pertenecen más al grupo de relaciones de tipo secundario. La razón y el pensamiento priman más en el Colegio de Abogados, en la Universidad, etc., pero en la familia, como en el grupo de amigos y en la pareja de enamorados, priman relaciones emocionales, y pasiones que la sociedad siempre intenta controlar.

A fines del siglo xx, la familia está cumpliendo un papel básico y difícil y con la menor capacitación que uno pudiera imaginar. Socializar a las nuevas generaciones implica que, de alguna manera, se ejerce presión y fuerza sobre los miembros que están entrando a participar en nuestra sociedad, para que se adecuen a las formas de la vida social; no nos olvidemos que la socialización es una lucha espantosa entre las fuerzas centrípetas, que ejerce el grupo para tener los individuos adentro y fuerzas centrífugas que ejerce el individuo para arrancarse del grupo. En este sentido, la familia adquiere o tiene, desde el punto de la teoría sociológica, la connotación de grupo totalitario porque tiene que formar al sujeto en todos los segmentos que es necesario formar un individuo: biológico, psicológico, moral, cultural y social. Se divide esta tarea de socialización en los grupos que se llaman segmentarios: la Universidad, por ejemplo, es segmentaria, se preocupa de un sector de la vida del sujeto que está con ellos, pero la familia tiene una responsabilidad integral, y ¿cuál es la capacitación que tiene la familia para ejercerla funcionalmente?

No nos olvidemos que el proceso de socialización, exige que la familia debe lograr en un período muy corto el control de los esfínteres; producir conductas éticas; producir el desarrollo psicobiológico. ¿Y cómo se consigue esto? si no hay un plan de vida, a lo mejor tendríamos un sujeto de 30 años que no controla sus

Octubre XII, 1987, en Dinamarca: Gottlieb y otros, 1987, han comprobado que los homicidios cometidos por pacientes mentales tienen como víctimas a miembros de la familia o conocidos, ver: Gottlieb, P.; Gabrielsen, G. y Kramp, p. 1987. Psychotic Homicides in Copenhagen from 1979 to 1983. En: Acta Psychiatrica Scandinavic, vol. 76, N³, September (pp. 285-292). Munksgaard, Copenhagen. 
esfínteres, o tendríamos sujetos de 25 a 30 años que no cumplen ninguna responsabilidad social porque la familia no tuvo el tiempo suficiente para capacitarlo o no ejerció las fuerzas necesarias para su domesticación.

Frente a estos desafíos, ¿cómo está organizada la familia? La familia puede considerarse, desde un punto de vista sociológico y psicológico social, como grupo nuclear con dos grandes polos: un polo instrumental, el padre, y un polo afectivoemocional, la madre, por otra parte están los hijos constituyendo otros polos. En esta forma de organización social se ejerce naturalmente todo el petitorio que la sociedad hace a la familia: que se constituya para producir seres humanos funcionales a la sociedad.

En este libre juego de fuerzas, de repente empiezan a provocarse algunos desequilibrios entre los polos, no se saben administrar conflictos que son de tipo natural y que de algún modo aparecen como crisis insoslayables, una de ellas es la crisis generacional que está planteada, tanto del punto de vista biológico como social, como enfrentamiento imposible de sostener para algunos y no como la definió Ortega y Gasset: una polémica ilustrada entre las generaciones donde tiene que haber un traslape y no una eliminación de una por la otra.

Situaciones que desde esta perspectiva, dificultan a veces el análisis del problema de la violencia, de la penalidad y el problema de la percepción del fenómeno. ¿Cuáles son las formas de fidelidad y de respeto entre los cónyuges, que deben primar, según sean los contextos socioculturales? ¿Cuáles son los criterios de obediencia? ¿Qué relaciones debe haber entre padres e hijos y entre esposos?

El respeto entre los anglosajones, específicamente entre los norteamericanos, es distinto al respeto que hay entre los hispanos. En el respeto de los hispanos prima mucho el amor, el temor, la reverencia. En los norteamericanos está más el sentido igualitario; yo soy un igual y por lo tanto, en ese sentido, me respeto y respeto ${ }^{4}$.

¿Cuáles son las conductas amorosas apropiadas y adecuadas que deben ejercitarse y que son funcionales o disfuncionales en nuestra familia? ¿El problema del cortejo y de las respuestas? Hoy día no podemos estar indiferentes ante la responsabilidad de los medios de comunicación social, que nos están mostrando múltiples expectativas o formas de conducta que no se cumplen en nuestro medio; pero que las personas las asumen y esperan que se den y al no repetirse, se sienten frustradas y dan lugar a conflictos y provocaciones familiares. Se advierte en Chile, todavía, una desprotección jurídica de la familia y los niños ante la televisión, cosa que no ocurre por ejemplo en Inglaterra ${ }^{5}$.

$4 \quad$ Televisión: Antecedentes sobre un tema polémico, Julio Munizaga, Departamento de Extensión Universitaria. Universidad de Chile, 1970

5 Hay estudios comparativos al respecto. 
Entre otros problemas está el manejo de la etapa de adolescencia, que es muy conflictiva; la destrucción de bienes de la vivienda, cómo se sancionan o castigan. Muchas veces, en la familia, el agresor castiga rompiendo los bienes o el agredido también lo hace y obtiene a su vez respuestas de violencia.

El tratamiento que le damos a la ancianidad, disfrazamos una fuerza bastante negativa sobre nuestros ancianos, al ponerlos en una casa de reposo; estamos destrozando nuestra familia, por distintas razones, pero hay también una agresión hacia el anciano, desde ese punto de vista.

Se ha mencionado aqui e insisto en que tiene que primar el criterio y el contexto de la filosofía de vida en un momento.

Un famoso educador que vivió en tiempos de la Roma antigua, creó una máxima que formó a muchos, incluso a algunos de nosotros, que "la letra, con sangre entra", pero en el siglo x।x Pestalozzi, dijo: "No. Hay que humanizar la educación". Estos criterios van marcando el estilo cultural ${ }^{6}$. Un profesor alemán llevó, como buen alemán, una rigurosa estadística de la cantidad de varillazos, golpes y castigos que había dado a sus alumnos: sumaban millones. En esa cultura, no era violencia para la sociedad ni para los sistemas educativos, ni lo era para la familia.

Tenemos tres millones y medio de educandos y deseábamos ligar esta situación al tema de violencia intrafamiliar. ¿Cuál es el daño que sufre la familia cuando los niños fracasan en sus estudios? ¿Las inculpaciones son justas? Qué responsabilidad tiene la vivienda como un espacio en que la familia tiene que resolver una enorme cantidad de situaciones y se rompen las ecuaciones incluso a nivel biológico, con espacios muy reducidos en donde esta interacción se acelera y por supuesto, el conflicto adquiere mayores dimensiones. Recientemente, una tesis del Departamento de Psicología, de la Universidad de Chile comprueba la relación, en Chile, entre hacinamiento humano y problemas como violencia con los niños y otras perturbaciones sociales, en viviendas populares?.

Esta visión rápida tiene la intención de demostrar la gran heterogeneidad y multicausalidad de la violencia intrafamiliar.

En perspectivas coincidentes es necesario analizar y sintetizar todas estas experiencias y estos enfoques. El tratamiento monocausal de la violencia intrafamiliar crea más problemas y dificulta las soluciones.

5 Las cifras fueron tomadas de Principios de Educación del profesor Roberto Munizaga A., Ed. Universitaria, 1976.

7 Juan C. Errázuriz G. y Maria I. Guerra L. Descripción de la Interacción Familiar y de la Conducta Espacial de la Familia, en Distintas Condiciones de Densidad (Estudio Descriptivo-Comparativo). Tesis Depto. de Psicología, U. de Chile, 1986. 
¿De qué forma la violencia ambiental, la frustración, la falta de expectativas, crean reacciones agresivas que se expresan en la vida intrafamiliar?

La verdad es que ésta es una respuesta que tendría que ser dada en conjunto con algún psicólogo o psicólogo social. Pero nosotros podemos decir que la frustración o la presión ambiental tiene una cuota de importancia en esto. No se sabe cuál, pero lo más común es la imitación de ciertas formas o ciertos ejercicios de violencia en los medios de comunicación o la pérdida de empleo, en que hay una frustración.

En la pérdida de empleo puede producirse la situación que el marido, el hombre de la casa, queda en una posición económica inferior frente a la mujer, en la mayoría de estas situaciones cuando persiste la situación de madre o esposa que provee y hombre que no provee, el matrimonio tiende a sufrir conflictos.

Si hay estadísticas sobre tendencias en la agresión intrafamiliar de los últimos diez años, creo que podrían estructurarse tendencias, pero están menoscabadas por fallas del denuncio. Indudablemente, que interesaría ir perfeccionando los registros.

¿Qué se entiende por "fuerza positiva"?

Fuerza positiva es aquella que se ejerce para desarrollar conductas necesarias y adecuadas y que no tienen efectos negativos en el sentido de no afectar el desarrollo biológico, psicológico o social del individuo. Por ejemplo, si un niño está inapetente y no quiere comer, al niño hay que abrirle la boca y darle comida; el padre no puede darse el lujo de dejarlo morir. Igual sucede muchas veces con el dueño de casa, el hombre que se le caen los brazos y no quiere ir a trabajar; la familia va a tener que ejercer fuerza y violencia y ponerlo en la puerta de calle a lo mejor o arrastrarlo hasta el trabajo.

Cuando el niño no quiere ir al colegio, más de una vez yo mismo fui levantado en vilo y llevado al colegio como una manifestación de fuerza, ante la cual no tenía posibilidad de defenderme.

Pero en todas estas situaciones, como dicen los juristas, tiene que haber un castigo proporcional a la falta; pero, además, tiene que tener la cualidad de ser un castigo con afecto, un castigo que no cause daños o lesiones que provoquen más problemas o situaciones como las que estamos viendo aquí.

¿Qué sabe un padre de familia, mal entrenado, acerca del daño que puede hacer a un hijo dándole un golpe en la cabeza, por ejemplo, como castigo o correctivo o causando lesiones en un ojo? Es decir, las partes del cuerpo donde se castiga también son determinadas un poco por la cultura, por la sociedad y por la costumbre. Golpes a los senos, por ejemplo, a una mujer, que después probablemente provocarán lesiones, en el colegio es frecuente que los niños se agredan lanzando 
golpes a los órganos sexuales, entre los hombres, causando a lo mejor lesiones que pueden tener efectos toda la vida; hay otras culturas en que pelean a cabezazos.

También se castiga cuando hay conductas sexuales inadecuadas; la misión de la familia es reforzar la conducta sexual, hermanos que se interesen en tener relaciones sexuales incestuosas, vulneran la moral de nuestra sociedad y tiene que sancionarse drásticamente, ¿cómo se hace? ¿Cuándo se llama la atención? si no hay castigo, alguna forma de presión, con el afecto de por medio, hace muy improbable que nosotros orientamos positivamente la conducta del sujeto. En resumen, la familia requiere de una cultura de la violencia o la presión positivas.

Muchas mujeres, muchas madres, se van a encontrar con que de la noche a la mañana tienen que asumir el rol instrumental: tienen que empezar a sancionar, a castigar, y nunca estuvieron preparadas para ello. Aquí desapareció el juez, el padre, el hombre que puede aplicar fuerza. ¿Cuánto saben de fuerza y cuánto pueden aplicar de fuerzas las madres? Creo que hoy día si viéramos, esto es una hipótesis, vamos a encontrar que hay una gran cantidad de lesiones que nos afectan, de madres a sus hijos. La madre, en forma descontrolada, no halla qué hacer para resolver una situación y no sabe castigar, quizás el hombre, "castigador más profesional", pueda aplicar mejor los castigos, lo que no significa que tenga el derecho a hacerlo en forma desmedida ni hacerlo en la forma y con los efectos que a nosotros nos preocupan aquí.

¿Qué cuota de responsabilidad le corresponde a un sistema político, ante la actitud agresiva de la gente frente a una determinada situación?

Creo que no hay sistema político que pueda evitar responsabilizarse de esto. La política es la ciencia de ejercitar el poder, de dirigir una sociedad; aquí se está apuntando a lo que se llaman las instituciones expresivo-integrativas. Es decir, indudablemente que un gobierno, un Estado tiene que idear o sostener o mantener mecanismos de control, que sean funcionales para que no ocurra violencia negativa, privilegiar las organizaciones de integración y de cohesión; por supuesto desarrollar organismos que se mencionan frecuentemente: tribunales especializados para la familia, por ejemplo. Nosotros estamos especializados en fragmentar la sociedad. Niños, jóvenes, hombres maduros, ancianos, etc., siguiendo taxonomías de clasificación clásicas. Pero el tribunal de problemas familiares, no existe. Tenemos tribunales de menores, que tienen una legislación que afecta a los padres en determinados casos, pero no analiza el contexto global de los problemas de la familia, donde deberían examinarse mejor todas estas situaciones.

¿Cuándo el sistema es agresivo en sí?

Éste también es un problema de percepción de valores y es relativo. Contestando, digo que es agresivo cuando la sociedad define qué es agresivo como tal y tiene consecuencias negativas; la agresividad se aprende. Hay una, natural, que el sujeto tiene y aprende la forma social, o la forma cultural de ejercitarla; claro que se imita, 
pero no todos los sujetos sometidos a agresividad, la aprenden y la imitan. Esa es una cuestión de interés general para la ciencia.

¿Por qué, si el instinto y el deseo sexual es tan fuerte, tan terriblemente fuerte desde el punto de vista instintivo, no hay más violencia en ese sentido?

Porque hay suficientes mecanismos de control, llamémoslo de conciencia o de presión para que no ocurra. Hay muchas cosas más que podríamos ejemplificar aquí, que son, instintivamente muy fuertes. Sin embargo, hay controles. Pero en algunos aspectos, en la sociedad, fallan los controles. El problema esencial es prevenir y prever, en vez de castigar la falta, se deben modificar algunas situaciones para evitar que estos daños se produzcan. 